\title{
Etiologie des infertilités secondaires de l'homme
}

\author{
Clément DARCQ, Jean HERMABESSIERE
}

Service d'Urologie Félix Guyon, Centre République, Clermont-Ferrand

\section{RÉSUMÉ}

Introduction : L'infertilité secondaire se définit comme l'impossibilité d'obtenir une nouvelle grossesse pour un couple ayant déjà procréer dans le passé.

Matériel et méthode : Sur une période de 16 ans, 49 patients sont venus consulter pour un problème d'infertilité secondaire d'origine masculine. Un bilan clinique et para-clinique a permis de conclure sur l'origine de l'infertilité.

Résultats : L'âge moyen de ces patients était de 33,6 ans (de 26 à 44 ans). Les enfants vivants obtenus auparavant dans ces couples étaient en moyenne de 1 par famille, représentés par une fille deux fois sur 3 , et un garçon une fois sur 3 . Les résultats obtenus chez ces patients montrent que l'infertilité est due à la présence d'une varicocèle pour 15 malades soit $31 \%$ des cas, la présence d'une infection pour 14 patients soit $29 \%$, et la présence d'une varicocèle associée à une infection dans 11 cas soit $22 \%$.

Conclusion : deux étiologies principales se dégagent dans le cadre de ce bilan. Cependant, certaines causes restent évidentes, comme les interventions chirurgicales avec ablation de la prostate ou des testicules, ou certains traitement cytotoxiques.

Mots clefs : infertilité secondaire, homme, varicocèle, infection

\section{INTRODUCTION}

La stérilité secondaire atteint des hommes en âge de procréer, ayant déjà eu un ou des enfants (ou grossesse) avec la même femme, et se trouvant dans l'incapacité d'obtenir une nouvelle grossesse. Ceci sous entend que la femme soit elle aussi en âge de procréer, qu'elle ait un bilan d'infertilité négatif, que le couple soit resté le même, et que les enfants existant dans le couple soient bien issus du père en question.

\section{MATÉRIEL ET MÉTHODE}

Sur une période de 16 ans, 49 patients sont venus consulter pour un problème d'infertilité secondaire. Tous les hommes ont été vus en consultation d'infertilité avec un spermogramme. Tous les patients ont été soumis à un interrogatoire, à la recherche de paternité antérieure, d'antécédents évocateurs, de prise de médicaments ou toxiques. Ils ont tous bénéficiés d'un examen clinique à la recherche d'une anomalie de l'appareil génito-urinaire, ou d'anomalies pouvant faire évoquer une pathologie endocrinienne. En fonction des données de l'examen clinique, des examens complémentaires ont été prescrits, tel qu'une échographie testiculaire à la recherche d'une varicocèle, des dosages biologiques hormonaux (Téstotéronémie, $\mathrm{FSH}, \mathrm{LH}, \mathrm{TSH}$, Prolactinémie), des examens bactériologiques (ECBU, spermogramme, spermoculture, prélèvement uréthral).

\section{Correspondance :}

Dr Jean HERMABESSIERE - Service d'urologie Félix Guyon, Centre République, 99 avenue de la République, 63000 Clermont-Ferrand. 


\section{RÉSULTATS}

L'âge moyen de ces patients était de 33,6 ans (de 26 à 44 ans). Parmi les antécédents de ces hommes, une infection urogénitale était présente dans 5 cas, des interventions pour cryptorchidie dans 4 cas, une varicocèle opérée pour 2 patients, un phimosis pour 2 , une cure de hernie inguinale pour 2 patients, une torsion du cordon spermatique pour 1 , et un cancer du testicule dans un cas.

Ces hommes étaient mariés (ou vivaient en couple) depuis en moyenne 8,7 ans. Leurs épouses étaient âgées en moyenne de 31,3 ans, et étaient donc plus jeunes que les hommes dans $90 \%$ des cas de 2 ans et 3 mois en moyenne. Les enfants vivants obtenus auparavant dans ces couples étaient en moyenne de 1 par famille, représentés par une fille deux fois sur 3 , et un garçon une fois sur 3 . Cinq familles avaient plus de 1 enfant, et dans ce cas, le nombre total d'enfant n'excédait pas 2.

Le nombre de grossesse n'ayant pas aboutit était de 12 pour 51 couples, soit en moyenne 0,23 grossesse par couple ou environ 1 interruption de grossesse pour 4 couples. Ces grossesses ont été prématurément interrompues pour des fausses couches spontanées dans 9 cas, 1 cas d'enfant mort-né, et 2 cas d'IVG. Pour les couples ayant dans leur antécédentes une grossesse interrompue prématurément, il s'agissait de la première grossesse pour 4 couples sur les 12 , soit $33 \%$.

Le désir d'avoir une deuxième enfant ou pour certain couple d'obtenir une deuxième grossesse remonte en moyenne à 2,4 ans, allant de 2 mois à 10 ans.

Une étiologie a pu être retenu au terme de ce bilan pour 44 patients soit dans $90 \%$ des cas.

Les résultats obtenus chez ces patients montrent que l'infertilité est due à la présence d'une varicocèle pour 15 malades soit $31 \%$ des cas, un antécédent d'infection pour 14 patients soit $29 \%$, et la présence d'une varicocèle associée à une infection dans 11 cas soit $22 \%$.

Parmi les patients atteints de varicocèle, le diagnostic a été clinique, ou fait dans tous les cas par une échographie. II faut noter qu'un patient présentait en même temps une ligature accidentelle du déférent droit suite à une chirurgie pour hernie inguinale droite, qu'un patient présentait en plus des problèmes du comportement sexuel pour lequel une prise en charge sexologique a été nécessaire, et enfin un troisième présentait une varicocèle gauche associée à une atrophie testiculaire homo latérale.
La notion d'infection a été portée sur les antécédents d'épididymite retrouvée à l'interrogatoire dans la plupart des cas.

Les autres causes plus rares retrouvées sont un cas d'azoospermie après radiothérapie pour le patient atteint de cancer du testicule, un patient porteur d'un hyperœstrogénisme, un autre atteint d'hyperprolactinémie, et un dernier atteint d'une cause immunologique, mise en évidence tout d'abord par la présence d'agglutinants sur le spermogramme, puis la présence d'anticorps antispermatozoïdes.

\section{DISCUSSION}

L'infertilité secondaire est un problème rencontré peu fréquemment en consultation, contrairement à l'infertilité primitive. Peu d'études ont été réalisée à ce sujet. Les quelques études mentionnant cette entité portent sur l'analyse de l'infertilité primaire ou secondaire sans faire de distinction.

Les principales étiologies semblent être la varicocèle, les antécédents d'infection et les traumatismes testiculaires [34]. Martin du Pan et Campana [22] ont étudié les facteurs étiologiques de stérilité primaire ou secondaire à propos de 350 cas. D'après eux, les causes acquises telles prostatites, varicocèle, ou causes autoimmunes représentaient $36,8 \%$ des cas, alors qu'une insuffisance testiculaire était retrouvée dans $23 \%$, une azoospermie obstructive dans $7,4 \%$, des facteurs environnementaux dans $12 \%$, et dans $19 \%$ des cas aucune cause n'était retrouvée.

L'infection est donc une des étiologies d'infertilité secondaire. Il est très difficile d'apporter des chiffres exacts quant à la responsabilité des infections dans l'apparition d'une stérilité. Dans notre étude, les infections des voies génito-urinaires sont responsables de $51 \%$ des infertilités secondaires.

II se produit au moment d'une infection une destruction de cellules souches, qui peut aboutir à une perte totale de la spermatogenèse si l'infection se prolonge sans traitement, ou si elle se répète. La qualité du sperme est souvent altérée, avec une augmentation de la viscosité, une baisse du volume, une baisse du $\mathrm{pH}$, une baisse de la concentration des substances secrétées par les glandes annexes, ne pouvant plus de ce fait assurer sa fonction de vecteur de spermatozoïdes. Une altération des diverses fonctions du sperme serait donc la cause principale d'infertilité associée souvent à une obstruction des voies excrétrices.

Les bactéries retrouvées sont très souvent pourvoyeuses de MST tels Chlamydia trachomatis, qui est la bactérie la plus souvent en cause dans les problèmes d'infertilité 
dans les régions industrialisées, ou Neisseria gonorrhoeae, qui est probablement l'agent le plus pourvoyeur d'obstacle sur les voies génitales [13]. Le mycoplasme (Uréaplasma urealyticum) qui est retrouvée dans 10 à $40 \%$ dans le sperme selon Keck et al. [17], serait également à l'origine de problème d'infertilité, puisqu'il paraît être en quantité supérieure dans la population d'hommes infertiles [39].

Les entérobactéries peuvent être également incriminées dans la survenue d'une infertilité. Villanova-Diaz et al. retrouvent la présence d'entérocoques dans $43 \%$ des spermes de patients infertiles, Escherchia coli dans $24 \%$, staphylocoque doré dans $19 \%$, et mycoplasme dans $14 \%$ [36].

Le bacille de Koch a été également reconnu comme pouvant engendrer des problèmes d'infertilité. Parfois, il est même le premier symptôme de la maladie, et le bilan d'infertilité peut conduire au diagnostic de tuberculose [19].

L'effet de la leucocytospermie reste très controversé, car les leucocytes jouent un rôle délétère sur le sperme, mais auraient également un rôle protecteur sur ce dernier [17].

Certains auteurs ont démontré la responsabilité du papilloma virus puisque la fréquence du papilloma était plus élevée dans la population infertile que dans la population générale [29]. Le virus du SIDA pourrait avoir un rôle néfaste direct sur les données du spermogramme [21], mais ceci reste à démontrer [6].

Les parasites seraient également pourvoyeur d'infertilité, principalement Trichomonas vaginalis, mais aussi les trypanosomiases, la leshmaniose, les amibes, la toxoplasmose, et le Plasmodium falciparum, agent du paludisme, ont été retrouvés dans les voies génitales masculines, responsables d'altérations testiculaires ou de l'axe hypothalamo-hypophysaire [24].

Le deuxième agent responsable d'infertilité serait la présence d'une varicocèle [3]. Le nombre de patients porteurs d'une varicocèle semble plus important dans une population d'hommes infertiles [10]. La prévalence de la varicocèle dans la population générale est de 10 à $15 \%$, et en cas d'infertilité secondaire, elle est évaluée entre $33 \%[14]$ et $45 \%[15,25]$. Cette entité reste cependant discutée et certains auteurs estiment que la prévalence du varicocèle ne diffère pas entre les hommes présentant une infertilité primaire par rapport aux hommes présentant un infertilité secondaire [15].

Cependant la varicocèle est responsable de certaines altérations du sperme et des spermatozoïdes, comme une oligo-asthéno-tératozoospermie $[3,10,11]$, mais là encore, un sperme normal peut être retrouvé assez fréquemment [14]. Pour Gorelick, la présence d'une varicocèle est significativement plus élevée chez des hommes atteints d'infertilité secondaire que des hommes atteints d'infertilité primaire [10]. La varicocèle entraîne donc des lésions évoluant progressivement vers une destruction des fonctions exocrines du testicule $[5,10]$. L'intervention sur les varicocèles chez des patients infertiles permet une bonne récupération de la fonction de reproduction pour $60 \%$ des patients $[3,10,11,16$, $18,32]$, et même une élévation du nombre de grossesse spontanée [3, 32].

Les troubles de la fertilité liés à la varicocèle seraient dus au reflux de substances toxiques provenant du rein ou de la surrénale, à de troubles hormonaux, à une hyperpression dans la veine funiculaire, à une hypoxie testiculaire due à la stase veineuse, et à des troubles de la régulation thermique au niveau des bourses $[8,25$, 32].

Un problème d'infertilité secondaire peut être observé dans les suites de dysrégulation endocrinienne d'origine centrale ou périphérique. En effet, toute affection tumorale primitive ou secondaire (adénome à prolactine, ou métastase), infectieuse (séquelle de méningite, ou neurosyphillis), traumatique, ou inflammatoire (sarcoïdose, histiocytose $X$, hémochromatose) de l'axe hypothalamo-hypophysaire peut entraîner un dérèglement de la sécrétion de $\mathrm{FSH}$ et $\mathrm{LH}$. Ces affections s'exprimeront par un hypogonadisme plus ou moins associé à d'autre déficit endocrinien. Enfin les troubles endocriniens périphériques, tel une hyper ou hypothyroïdie [26], ou un hypercorticisme [20] peuvent également entraîner une infertilité secondairement. L'obésité peut être le seul facteur étiologique d'une infertilité secondaire, notamment aux Etats-Unis, par réduction de la téstostéronémie [4].

Certaines affections urologiques peuvent également engendrer une infertilité secondaire. Tout d'abord, la chirurgie du pelvis, avec des atteintes du plexus nerveux hypogastrique, utilisée dans les prostatectomies radicales, ou les résections antérieures du rectum, sont pourvoyeuses d'impuissance, et d'absence d'éjaculation. Ensuite, les résections de prostate ou adénomectomies par voie haute entraînent souvent une éjaculation rétrograde. Enfin, les lésions traumatiques des testicules peuvent conduire à une castration ou à une atrophie testiculaire uni ou bilatérale, ou à la formation d'anticorps anti-spermatozoïdes à cause de la rupture de la barrière hémato-spermatique. Une contusion ou une plaie des déférents sera susceptible d'entraîner une fibrose et une sténose de celui-ci. Un traumatisme de la verge pourra être responsable d'une dysfonction érectile rendant les rapports sexuels impossible. 
Les tumeurs du testicule jouent obligatoirement un rôle néfaste sur la fertilité à cause d'une castration obligatoire, puis en raison des traitements complémentaires souvent indispensables (curage ganglionnaire, radiothérapie, ou chimiothérapie). La torsion du cordon spermatique est bien entendu un facteur pourvoyeur de stérilité par les lésions qu'une torsion peut entraîner au niveau d'un testicule, mais aussi par des anomalies constitutionnelles qui auraient été observés dans le testicule controlatéral à la torsion [1]. Par ailleurs des phénomènes immunologiques seraient également présents pour expliquer la stérilité chez ces patients, en raison de l'effraction de la barrière hémato-spermatique lors d'épisodes de sub-torsion rencontrés dans le passé de ces patients [2].

Des affections générales peuvent avoir également des répercutions au niveau du tractus génital, et altérer la fonction reproductive. L'hypertension, l'infarctus du myocarde peuvent altérer la fonction testiculaire par une dys-régulation endocrinienne centrale avec chute de la téstostéronémie [7, 38]. II existe également des troubles de la régulation hormonale d'origine hypothalamique pour les patients suivis pour une insuffisance rénale, probablement en rapport avec un effet toxique de l'urée sur l'hypothalamus [12].

Enfin, il semble qu'un certain nombre de causes environnementales puisse provoquer des problèmes d'infertilité secondaires. L'exposition de certains agents chimiques, comme le dibromochloropropane [37], les métaux lourds (plomb, cadmium, mercure) [40] ou certains composés organiques [27,33], et les radiations ionisantes $[30,35]$ peuvent avoir des effets nocifs sur la reproduction. Certaines intoxications chroniques peuvent également avoir de tels effets : l'alcool [28], et certaines drogues "dures" dans $12 \%$ des cas [23], la cigarette [31], et enfin certains psychotropes [9].

\section{CONCLUSION}

L'infertilité secondaire au sein d'un même couple est un phénomène rare et encore mal étudié. Certaines causes sont évidentes, comme les interventions chirurgicales avec ablation de la prostate ou des testicules, ou certains traitement cytotoxiques.

Les autres causes, dominant en fait largement cette pathologie, sont la présence d'une varicocèle, et les antécédents d'infection du tractus uro-génital. Ces deux causes sont à rechercher en priorité lors de l'examen clinique, qui prend ici toute sa valeur. Cela ne fait que confirmer l'importance de ces deux étiologies dans l'infertilité primaire et secondaire.

\section{REFERENCES}

1. ANDERSON J.B., WILLIAMSON R.C.N. : The fate of the human testes following unilateral torsion of the spermatic cord. Br. J. Urol., 1986, $73: 847-849$.

2. ANDERSON J.B., WILLIAMSON R.C.N. : Fertility after torsion of the spermatic cord. Br. J. Urol., 1990, $65: 225-230$.

3. BELKERA.M. : The varicoclele and male infertility. Urol. Clin. North Am., 1981, $8: 41-51$.

4. BRAY G.A. : Obesity and reproduction. Hum. Reprod., 1997, 12 : 26-32.

5. CHEVAL M.J., PURCELL M.H. : Deterioration of semen parameters over time in men with untreated varicocele : evidence of progressive testicular damage. Fertil. Steril., 1992, 57 : 12891293.

6. CRITTENDEN J.A., HANDELSMAN D.J., STEWART G.J. : Semen tract inflammation and male virus infection. Fertil. Steril., 1992, 57 : 1294-1299.

7. DONG Q., HAWER F., MCWILLIAM D., BANGAH M., BURGER H., HANDELSMAN D.J. : Circulating inhibin and testosterone levels in men with critical illness. Clin. Endocrinol., 1992, 36 : 399404.

8. FUJISAWA M., YOSHIDA S., KOJIMA K., KAMIDONO S. : Biochemical changes in testicular varicocele. Arch. Androl., 1989, $22: 149-159$

9. GETZOFF P.L. : Psychotropic drug induced male infertility : a case report. Infertility, 1978, $1: 53-58$.

10. GORELICK J.L., GOLDSTEIN M. : Loss of fertility in men with varicocele. Fertil. Steril., 1993, $59: 613-161$.

11. HAFEZ B. : Recent davances in clincal/molecular andrology. Arch. Androl., 1998, $40: 187-210$.

12. HANDELSMAN D.J. : Hypothalamic-pituitary gonadal dysfunction in chronic renal failure, dialysis, and renal transplantation. Endocr. Rev., 1985, $6:$ 151-182.

13. HANDSFIELD H.H., LIPMAN T.O., HARNISH J.B., TRONCAE., HOLMES K.K. : Asymptomatic gonorrhea in men : diagnosis natural course, prevalence and significance. N. Eng. J. Med., 1974, $290: 117-122$.

14. HIROKAWA M., IWAMOTO T., IWASAKI A., YAMAZAKI A., ASAKURAS., MATSUSSHITAK. : Clinical observations on fertile males with varicocele. Eur. Urol., 1989, $16: 23-27$.

15. JAROW J.P., COBURN M., SIGMAN M. : Incidence of varicoceles in men with primary and secondary infertility. Urology, 1996, 47: 73-76.

16. KASS E.J., BELMAN A.B. : reversal of testicular growth failure by varicocele ligation. J. Urol., 1987, $137: 475-476$.

17. KECK C., GERBER-SCHAFER C., CLAD A., WILHLEM C., BRECKWOLDT $M$. : Seminal tract infections : impact on male fertility and treatment options. Hum. Reprod. Update, 1998, 4 : 891-903.

18. KIBAR Y., SECKIN B., ERDURAN D. : The effects of subinguinal varicocelectomy on Kruger morphology and semen parameters. J. Urol., 2002, 168 : 1071-1074.

19. LUBBE J., RUEF C., SPIRIG W., DUBS M., SIGG C. : Infertility as the first symptom of male genitourinary tuberculosis. Urol. Int., 1996, 56 : 204-206.

20. LUTON J.P., THIEBLOT P., VALCKE J.C., MAHOUDEAU J.A., BRICAIRE H. : Reversible gonadotrophin deficiency in male Cushing's disease. J. Clin. Endoncrinol. Metab., 1977, 45 : 488495. 
21. MARTIN P.M.V., GRESSENHUET G., HERVE V.M., RENOM G., STENMAN G., GEORGES A.J. : Decreased number of spermatozoa in HIV infected individuals. AIDS, 1991, 6, 130.

22. MARTIN DU PAN R.C., CAMPANAA. : Etiologie de 350 cas de stérilité masculine. Effet de divers traitements sur la qualité du sperme et analyse de leur rôle dans la survenue de 100 grossesses. Andrologie, 1997, $7:$ 199-211.

23. MARTIN DU PAN R.C., BISCHOF P., CAMPANAA. : Relationship between etiological factors and total motile sperm count in 350 infertile patients. Arch. Androl., 1997, 33 : 197-210.

24. MARTINEZ-GARCIA F., REGADERA J., MAYER R., SANCHEZ S., NISTAL M. : Protozoan infections in the male genital tract. J. Urol., 1996, $156:$ 339-349.

25. NAUGHTON C.K., NANGIAA.K., AGARWALA. : Patholphysiology of varicocele in male infertility. Hum. Reprod. Update, 2001, 7 : 473-481.

26. O'BRIEN I.A.D., LEWIN I.G., O'HARE J.P., CORRALL R.J.M. : Reversible male subfertility due to hyperthyroidism. Br. Med. J., 1982, $285: 691$.

27. OLIVA A., SPIRA A., MULTIGNER L. : Contribution of environmental factors to the risk of male infertility. Hum. Reprod., 2001, $16: 1768-1776$.

28. PAJRINEN J.T., KARHUNEN P.J. : Spermatogenic arrest and 'Sertoli cell-colony" syndrome-common alcohol-induced disorders of the human testis. Int. J. Androl., 1994, $17: 292-299$.

29. PAKENDORF U.W., BORNMAN M.S., DU PLESSIS D.J. : Prevalence of human papilloma virus in men attending the infertility clinic. Andrologia, 1998, $30: 11-14$.

30. ROWLEY M.J., LEACH D.R., WARNER G.A., HELLER C.G. : Effects of graded doses of ionizing radiation on the human testis. Radiat. Res., 1974, $59: 665-678$.

31. SALEH R.A., AGARWAL A., SHARMA R.K., NELSON D.R., THOMAS A.J. : Effetcs of cigarette smoking on levels of seminal oxidative stress in infertile men : a prospective study. Fertil. Steril., 2002, $78:$ 491-499.

32. SCHOOR R.A., ELHANBLY S.M., NIEDERBERGER C. : The pathophysiology of varicocele-associated male infertility. Curr. Urol. Rep., 2001, 2, 432-436.

33. SHARPE R.M. : Declining sperm counts in men-is there an endocrine cause ? : J. Endocrinol., 1993, $136:$ 357-360.

34. THONNEAU P., BUJAN L. : Epidémiologie des infertilités masculines. Rev. Prat., 1993, 43 : 936-940.

35. THONNEAU P. : Fertility and environment: a new challenge. Contracept Fertil. Sex., 1993, 21 : 639-641.

36. VILLANOVA-DIAZ C.A., FLORES-REYES G.A., BELTRANZUNIGA M., ECHAVARRIA-SANCHEZ M., ORTIZ-IBARRA F.J., ARREDONDO-GARCIA J.L. : Bacteriospermia and male infertility : a method for increasing the sensitivity of semen culture. Int. J. Fertil. Womens Med., 1999, 44 : 198-203.

37. WHORTON D., KRAUSS R.M., MARSHALL S., MILBY T.H. : Infertility in male pesticide workers. Lancet, 1977, $2: 1259-1261$.

38. WOOLF P.D., HAMIL R.W., MCDONALD J.V., LEE L.A., KELLY M. : Transient hypogonadotrphic hypogonadism caused by critical illness. J. Clin. Endocrinol. Metab., 60 : 444-450, 1985.

39. XU C., SUN G.F., ZHU Y.F., WANG Y.F. : The correlation of ureaplasma urealyticum infection with infertility. Andrologia, 1997, $29: 219-226$.

40. XUEZHI J., YOUXIN L., YILAN W. : Studies of lead exposure on reproductive system : A review of work in China. Biomed. Environ. Sci., 1992, 5 : 266-275.

\author{
ABSTRACT \\ Aetiology of male secondary infertility \\ Clément DARCQ, Jean HERMABESSIERE
}

Introduction: Secondary infertility is defined as the inability to achieve a new pregnancy for a couple that has been able to procreate in the past.

Material and methods: Over a 16-year period, 49 patients consulted for male secondary infertility. Clinical, laboratory or ultrasound assessment demonstrated the cause of infertility.

Results: These patients had a mean age of 33.6 years (range: 26 to 44 years). These couples had an average of 1 living child per family, corresponding to a girl in twothirds of cases and a boy in one-third. In these patients, infertility was due to the presence of varicocele in 15 patients (31\% of cases), infection in 14 patients $(29 \%)$, and varicocele associated with infection in 11 cases $(22 \%)$.

Conclusion: This assessment revealed two main aetiologies, but some causes remain obvious, such as surgery with resection of the prostate or testicles, or certain cytotoxic therapies.

Key-words: secondary infertility, men, varicocele, infection 